\title{
Wireless Multicasting with Diversity Combining Techniques
}

\author{
Rosni Sayed ${ }^{1}$, Md Asaduzzaman Shobug ${ }^{2}$ \\ ${ }^{1,2}$ (Electrical and Electronic Engineering, Pabna University of Science \& Technology, Bangladesh)
}

\begin{abstract}
We consider Rayleigh fading SIMO multicasting wireless network. At first, we characterize the channel capacity. Then the closed form expressions of Probability Density Function (PDF), Cumulative Distribution Function (CDF) and Complementary Cumulative Distribution Function (CCDF) of the capacity are derived by applying two diversity combining techniques, such as Selection Combining (SC) and Maximal Ratio Combining (MRC). Finally we present a comparison between these two types of diversity combining techniques.
\end{abstract}

Keywords: $C C D F, C D F$, diversity combining techniques, MRC, Multicasting wireless network, Outage Probability, SC

\section{Introduction}

Multicasting has become more popular way of supporting group communication than broadcasting because it allows transmission to multiple destinations using fewer network resources. The information is delivered to each of the links only once, and copies are created when the links to the destinations split, thus creating an optimal distribution path. Multicast over wireless networks is a fundamental communication function as well as a challenging goal. The goal is to substantially reduce bandwidth and the power consumed. Although multicasting ensures better utilization of communication resources, channel fading is still a big threat for sound wireless communication. There are two techniques to combat the effect of fading. They are

- Transmitter Power Control

- Diversity Technique

In Transmitter Power Control method, there are two major drawbacks. First, the transmitter requires a dynamic range. Second, channel information has to be fed back from the receiver to the transmitter. So, diversity combining technique is a good alternative to transmitter power control method. In receiver diversity the independent fading paths associated with multiple receive antennas are combined to obtain a resultant signal that is then passed through a standard demodulator. The combining can be done in several ways which vary in complexity and overall performance. Diversity Combining Techniques are applied to combine the multiple received signals of a diversity reception device into a single improved signal to get proper diversity benefit [1]. The combing system should be in such a manner that improves the performance of the communication system like the signal-to-noise ratio (SNR) or the power of received signal. Mainly, the combining should be applied in reception; however it is also possible to apply in transmission.

Several Combining Techniques are listed below

* Selection Combining (SC)

* Maximal Ratio Combining (MRC)

* Threshold or Switch and Stay Combining (SSC)

Recently, there has been huge interest on diversity combining techniques, several work have been done on this topic. D. G. Brennan analysed different diversity combining techniques in practical use ${ }^{[2]}$. Luu Pham Tuyen et. Al applied combining and decoding technique at the receivers to reduce fading effect of MIMO (Multiple Input Multiple Output) channel ${ }^{[3]}$. Jorg Nuchell et. al evaluated results of different linear diversity combining techniques. They showed that the use of multiple receive antennas in combination with adequate signal combining can improve the reliability of communication system even under poor SNR ${ }^{[4]}$. A. Arokiamarie Johnsi, V. Saminadan proposed diversity combining techniques in Free space Optical (FSO) communication system to mitigate the harmful effect of atmospheric turbulence ${ }^{[5]}$. Sari khatalin, John Fonseka derived closed form expressions for the capacity of dual branch maximum ratio combining, equal gain combining, selection combining and switching and stay combining over correlated Nakagami-m fading channels [6].

In this paper, we consider a multicasting Rayleigh fading system. We derive closed form expression of outage probability of channel and CCDF of multicast capacity by applying diversity combining techniques.

Rest of the paper is organized as follows. In section II, system model is shown. Chapter III, IV and V describes the formulation of PDF, outage probability and CCDF applying two different diversity combining 
techniques. In section VI, the numerical result is represented graphically. Finally we draw a conclusion of the work in section VII.

\section{System Model}

This paper considers a multicasting scenario as shown in Figure 1. Here a single antenna transmitter communicates with $M$ receivers. Each receiver is equipped with $n_{R}$ antennas. The total system is considered under Rayleigh fading. The channel coefficient between the source and the $\mathrm{i}^{\text {th }}$ receiver is $h_{i}$, where, $i=$ $1,2,3 \ldots \ldots, M$. Let $x$ denotes the transmitted signal. The received signals at the $\mathrm{i}^{\text {th }}$ receiver given by (1), that is:

$$
\boldsymbol{y}_{\boldsymbol{i}}=\boldsymbol{h}_{i} x+\boldsymbol{z}_{i}
$$

Where $\boldsymbol{z}_{i} \sim \tilde{\mathcal{N}}\left(0, N_{m_{0}} I_{n_{R}}\right)$ is the Gaussian noise, imposed on the $\mathrm{i}^{\text {th }}$ receiver.

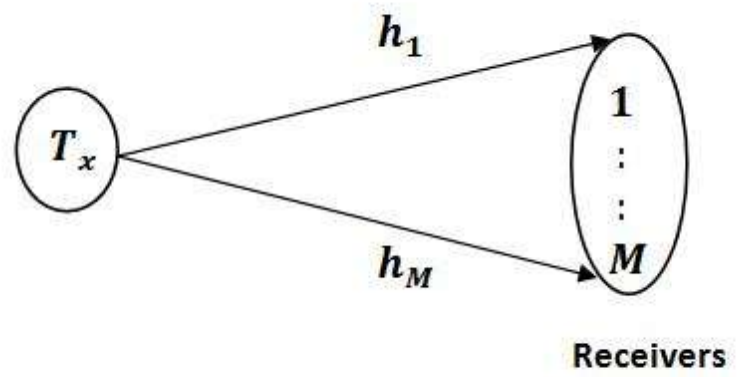

Figure 1: System Model

From (1) the received signal at the $i^{\text {th }}$ user is given by

\section{Multicast Capacity}

Mutual information at $i^{\text {th }}$ user is

$$
\boldsymbol{y}_{\boldsymbol{i}}=\boldsymbol{h}_{i} x+\boldsymbol{z}_{i}
$$

$$
I\left(x ; y_{i}\right)=h\left(y_{i}\right)-h\left(\mathbf{z}_{i}\right)
$$

Here $h($.$) denotes entropy. Let the variance of x$ is given by $Q_{x}=\mathbb{E}\left(\mathrm{xx}^{\dagger}\right)=\mathrm{P}$, where $\mathbb{E}($.$) and (.)^{\dagger}$ denote the expectation and conjugate transpose operations, respectively. Now, co-variance of received signal can be derived as :

$$
\begin{aligned}
R_{y_{i}}=\mathbb{E}\left(\boldsymbol{y}_{\boldsymbol{i}}, \boldsymbol{y}_{\boldsymbol{i}}^{\dagger}\right) & =\mathbb{E}\left\{\left(\boldsymbol{h}_{i} x+\boldsymbol{z}_{i}\right)\left(\boldsymbol{h}_{i} x+\boldsymbol{z}_{i}\right)^{+}\right\} \\
& =\mathbb{E}\left\{\left(\boldsymbol{h}_{i} x+\boldsymbol{z}_{i}\right)\left(x^{+} \boldsymbol{h}_{i}^{+}+\boldsymbol{z}_{i}^{+}\right)\right\} \\
& =\mathbb{E}\left(\boldsymbol{h}_{i} x x^{+} \boldsymbol{h}_{i}^{+}+\boldsymbol{z}_{i} \mathbf{z}_{i}^{+}\right) \\
& =\boldsymbol{h}_{i} \mathbb{E}\left(x x^{+}\right) \boldsymbol{h}_{i}^{+}+\mathbb{E}\left(\mathbf{z}_{i} \mathbf{z}_{i}^{+}\right) \\
& =\boldsymbol{h}_{i} P \boldsymbol{h}_{i}^{+}+N_{m_{o}} \boldsymbol{I}_{n_{R}}
\end{aligned}
$$

Similarly, covariance of noise signal is given by:

$$
R_{z_{i}}=\mathbb{E}\left(\mathbf{z}_{i} \mathbf{z}_{i}^{+}\right)=N_{m_{o}} \boldsymbol{I}_{n_{R}}
$$

Hence, the entropy of $\boldsymbol{y}_{\boldsymbol{i}}$ is given by:

$$
h\left(y_{i}\right)=\log _{2} \operatorname{det}\left(\pi e R_{y_{i}}\right)=\log _{2} \operatorname{det}\left[\pi e\left(\boldsymbol{h}_{i} P \boldsymbol{h}_{i}^{+}+N_{m_{o}} \boldsymbol{I}_{n_{R}}\right)\right]
$$

Similarly, the entropy of $\boldsymbol{z}_{i}$ is given by:

$$
h\left(\mathbf{z}_{i}\right)=\log _{2} \operatorname{det}\left(\pi e R_{\mathbf{z}_{i}}\right)=\log _{2} \operatorname{det}\left(\pi e N_{m_{o}} \boldsymbol{I}_{n_{R}}\right)
$$

Hence the mutual information at the $i^{\text {th }}$ user is given by:

$$
\begin{aligned}
I\left(x ; y_{i}\right)=\log _{2} \frac{\operatorname{det}\left(\pi e R_{y_{i}}\right)}{\operatorname{det}\left(\pi e R_{\mathbf{z}_{i}}\right)} & =\log _{2} \frac{\operatorname{det}\left[\pi e\left(\boldsymbol{h}_{i} P \boldsymbol{h}_{i}^{+}+N_{m_{o}} \boldsymbol{I}_{n_{R}}\right)\right]}{\operatorname{det}\left(N_{m_{o}} \boldsymbol{I}_{n_{R}}\right)}=\log _{2} \frac{\operatorname{det}\left[\pi e\left(\boldsymbol{h}_{i} P \boldsymbol{h}_{i}^{+}+N_{m_{o}} \boldsymbol{I}_{n_{R}}\right)\right]}{\operatorname{det}\left(N_{m_{o}} \boldsymbol{I}_{n_{R}}\right)} \\
& =\log _{2}\left(1+\frac{P}{N_{m_{o}}}\left\|\boldsymbol{h}_{i}\right\|^{2}\right)
\end{aligned}
$$

Multicast capacity of $i^{\text {th }}$ user is given by:

$$
C_{m}=\log _{2}\left(1+\frac{P}{N_{m_{o}}} \min _{1<i<M}\left\|\boldsymbol{h}_{i}\right\|^{2}\right)=\log _{2}\left(1+\theta_{1} \min _{1 \leq i \leq M} \gamma_{m_{i}}\right),
$$

where $\theta_{1}=\frac{P}{N_{m_{o}}}$ and $\min _{1<i<M} \gamma_{m_{i}}$ is the minimum instantaneous SNR among all the users.

Let the PDF of $\gamma_{m}$ is given by $f_{\gamma_{m}}\left(\gamma_{m_{i}}\right)$, Then the distribution of instantaneous SNRs at $i^{\text {th }}$ user can be expressed as:

$$
f_{d \min }\left(\gamma_{m_{i}}\right)=M f_{\gamma_{m}}\left(\gamma_{m_{i}}\right)\left[1-F_{\gamma_{m}}\left(\gamma_{m_{i}}\right)\right]^{M-1}
$$


The cumulative distribution function (CDF) of $\gamma_{m_{i}}$ can be derived as:

$$
F_{\gamma_{m}}\left(\gamma_{m_{i}}\right)=\int_{0}^{\gamma_{m}} f_{\gamma_{m}}\left(\gamma_{m_{i}}\right) d \gamma_{m_{i}}
$$

\section{Proposition 3.1:}

Let the probability density function of $\mathrm{x}$ is denoted by $\mathrm{f}(\mathrm{x})$. Then the probability density function of $\mathrm{C}=$ $\log _{\mathrm{e}}(1+\theta \mathrm{x})$ is given by,

\section{Proof:}

$$
q(c)=\frac{e^{c}}{\theta} f\left(\frac{e^{c}-1}{\theta}\right)
$$

We have, $C=\log _{e}(1+\theta x)$. The probability density function of $C$, can be written as

$$
q(c)=\int \delta\left(C-\log _{e}(1+\theta x)\right) f(x) d x
$$

The following mathematical facts have been used for this proof [13];

i) $\quad \delta(f(x))=\sum_{l} \frac{\delta\left(x-x_{l}\right)}{\left|\frac{d f}{d x}\right|_{x_{l}}}$, where $x_{l}$ are the zeros of $f(x)$, i.e. $f\left(x_{l}\right)=0$;

ii) $\quad \int_{a}^{b} \delta\left(x-x_{1}\right) \delta\left(x-x_{2}\right) d x=\delta\left(x_{1}-x_{2}\right)$ for $a<x_{1}, x_{2}<b$; and

iii) $\quad \int_{V} f(x) \delta\left(x-x_{o}\right) d x=\left\{\begin{array}{c}f\left(x_{0}\right), x_{o} \in V \\ 0, \text { otherwise }\end{array}\right.$

Assuming $\quad \kappa=1+\theta x$, we have $f(\kappa)=C-\log _{e} \kappa \quad$ and $\quad f^{\prime}(\kappa)=-\frac{1}{\kappa}$. Now from $f(\kappa)=0, \kappa=e^{C}$ and $\left.f^{\prime}(k)\right|_{\kappa=e^{C}}=-\frac{1}{e^{c}}$. Using the above mathematical facts and from (13), we have

Where, $\delta($.$) is a delta function.$

$$
\begin{aligned}
q(c) & =\int e^{C} \delta\left(1+\theta x-e^{C}\right) f(x) d x \\
& =e^{c} \int \delta\left(\theta\left(x-\frac{e^{C}-1}{\theta}\right)\right) f(x) d x \\
& =\frac{e^{c}}{\theta} \int \delta\left(x-\frac{e^{C}-1}{\theta}\right) f(x) d x \\
& =\frac{1}{|d|} \delta(z)=\frac{e^{c}}{\theta} f\left(\frac{e^{C}-1}{\theta}\right),
\end{aligned}
$$

\subsection{MRC Diversity:}

The pdf of instantaneous received SNR $\gamma_{m_{i}}$ at the MRC output of the $\mathrm{i}^{\text {th }}$ user can be expressed as [1]:

$$
f_{\gamma_{i}}^{M R C}\left(\gamma_{m_{i}}\right)=\frac{\gamma_{m_{i}}^{n_{R}-1}}{\left(n_{R}-1\right) ! \gamma_{m_{o}}^{n_{R}}} e^{-\frac{\gamma_{m_{i}}}{\gamma_{m_{o}}}}
$$

Here, $\gamma_{m_{o}}$ is average SNRs per symbol at the user. The cumulative distribution function (CDF) of $\gamma_{m_{i}}$ can be derived as:

Using the identity, [7, eq. 3.351.1 \& eq. 3.351.2],

$$
F_{\gamma_{i}}^{M R C}\left(\gamma_{m_{i}}\right)=\int_{0}^{\gamma_{m}} f_{\gamma_{m}}^{M R C}\left(\gamma_{m_{i}}\right) d \gamma_{m_{i}}
$$

$$
\begin{aligned}
F_{\gamma_{i}}^{M R C}\left(\gamma_{m_{i}}\right) & =\frac{1}{\left(n_{R}-1\right) ! \gamma_{m_{o}}^{n_{R}}}\left[\frac{\left(n_{R}-1\right) !}{\left(\frac{1}{\gamma_{m_{o}}}\right)^{n_{R}}}-\left(\frac{1}{\gamma_{m_{i}}}\right)^{-n_{R}} \times \frac{1}{\left(n_{R}-1\right) !} \Gamma\left(n_{R}, \frac{\gamma_{m_{i}}}{\gamma_{m_{o}}}\right)\right] \\
& =1-\frac{\Gamma\left(n_{R}, \frac{\gamma_{m_{i}}}{\gamma_{m_{o}}}\right)}{\left(n_{R}-1\right) !}
\end{aligned}
$$

Now putting the value of (10) and (11) into (8) the PDF of the minimum instantaneous SNR is given by:

Using the proposition 3.1, the PDF of the capacity can be derived as :

$$
f_{d_{\min }}^{M R C}\left(\gamma_{m_{i}}\right)=M \frac{\gamma_{m_{i}}^{n_{R}-1}}{\left(n_{R}-1\right) ! \gamma_{m_{o}}^{n_{R}}} e^{-\frac{\gamma m_{i}}{\gamma m_{o}}} \times\left[1-\left(1-\frac{\Gamma\left(n_{R} \frac{\gamma m_{i}}{\gamma m_{o}}\right)}{\left(n_{R}-1\right) !}\right)\right]^{M-1}
$$

$$
q^{M R C}\left(C_{m}\right)=\frac{e^{C_{m}}}{\theta_{1}} f_{d_{\min }}^{M R C}\left(\frac{e^{C_{m}}-1}{\theta_{1}}\right)
$$




$$
\begin{aligned}
& =\frac{e^{C_{m}}}{\theta_{1}} M \frac{\left(\frac{e^{C_{m}}-1}{\theta_{1}}\right)^{n_{R}-1}}{\left(n_{R}-1\right) !^{M} \gamma_{m_{o}}^{n_{R}}} e^{-\frac{e^{C_{m}}-1}{\gamma_{m_{o}} \theta_{1}}}\left[\Gamma\left(n_{R}, \frac{e^{C_{m}}-1}{\gamma_{m_{o}} \theta_{1}}\right)\right]^{M-1} \\
& =e^{C_{m}} M \frac{\left(e^{C_{m}}-1\right)^{n_{R}-1}}{\left(n_{R}-1\right) !^{M} \gamma_{m_{o}}^{n_{R}}} e^{-\frac{e^{C_{m}-1}}{\gamma_{m_{o}}}}\left[\Gamma\left(n_{R}, \frac{e^{C_{m}-1}}{\gamma_{m_{o}}}\right)\right]^{M-1}
\end{aligned}
$$

considering $\theta=1$ for simplicity.

\subsection{SC Diversity}

Then the pdf of instantaneous received SNR $\gamma_{m}$ at the MRC output of users can be expressed as [1]

$$
f_{\gamma_{m_{i}}}^{S C}\left(\gamma_{m_{i}}\right)=\frac{n_{R}}{\gamma_{m_{o}}}\left[1-e^{-\frac{\gamma_{m_{i}}}{\gamma_{m_{o}}}}\right]^{n_{R}-1} e^{-\frac{\gamma_{m_{i}}}{\gamma_{m_{o}}}}
$$

The cumulative distribution functions (CDF) of $\gamma_{m_{i}}$ can be derived as

$$
\begin{array}{r}
F_{\gamma_{m_{i}}}^{S C}\left(\gamma_{m_{i}}\right)=\int_{0}^{\gamma_{m}} f_{\gamma_{m_{i}}}^{S C}\left(\gamma_{m_{i}}\right) d \gamma_{m_{i}} \\
=\int_{0}^{\gamma_{m}} \frac{n_{R}}{\gamma_{m_{o}}}\left[1-e^{-\frac{\gamma_{m_{i}}}{\gamma_{m_{o}}}}\right]^{n_{R}-1} e^{-\frac{\gamma_{m_{i}}}{\gamma_{m_{o}}}} d \gamma_{m_{i}} \\
\text { Let, } \quad 1-e^{-\frac{\gamma_{m_{i}}}{\gamma_{m_{o}}}}=z \\
\Rightarrow \frac{1}{\gamma_{m_{o}}} e^{-\frac{\gamma_{m_{i}}}{\gamma_{m_{o}}}} d \gamma_{m_{i}}=d z
\end{array}
$$

For, $\gamma_{m_{i}}=0, z=0$ and for $\gamma_{m_{i}}=\gamma_{m_{i}{ }^{\prime}} z=1-e^{-\frac{\gamma_{m_{i}}}{\gamma_{m_{o}}}}$.

$$
\begin{aligned}
\therefore F_{\gamma_{i}}^{S C} & \left(\gamma_{m_{i}}\right)=\int_{0}^{1-e^{-\frac{\gamma_{m_{i}}}{\gamma m_{o}}}} n_{R} z^{n_{R}-1} d z \\
& =\left[\frac{n_{R} z^{n_{R}}}{n_{R}}\right]_{0}^{1-e^{-\frac{\gamma_{m_{i}}}{\gamma m_{o}}}} \\
& =\left(1-e^{-\frac{\gamma_{m_{i}}}{\gamma_{m_{o}}}}\right)^{n_{R}}
\end{aligned}
$$

Using (14) and (15) into (8) we get:

$$
f_{d_{\text {min }}}^{S C}\left(\gamma_{m_{i}}\right)=M \frac{n_{R}}{\gamma_{m_{o}}}\left[1-e^{-\frac{\gamma_{m_{i}}}{\gamma m_{o}}}\right]^{n_{R}-1} e^{-\frac{\gamma m_{i}}{\gamma m_{o}}}\left[1-\left(1-e^{-\frac{\gamma m_{i}}{\gamma m_{o}}}\right)^{n_{R}}\right]^{M-1}
$$

Using proposition 3.1, the pdf of $C_{m}$ can be determined as

$$
\begin{gathered}
q^{S C}\left(C_{m}\right)=\frac{e^{C_{m}}}{\theta_{1}} f_{d_{\min }}^{S C}\left(\frac{e^{C_{m}}-1}{\theta_{1}}\right) \\
=e^{C_{m}} M \frac{n_{R}}{\gamma_{m_{o}}}\left[1-e^{-\frac{e^{C_{m}}-1}{\gamma_{m_{o}}}}\right]^{n_{R}-1} e^{-\frac{e^{C_{m}-1}}{\gamma_{m_{o}}}} \times\left[1-\left(1-e^{-\frac{e^{C_{m}-1}}{\gamma_{m_{o}}}}\right)^{n_{R}}\right]^{M-1}
\end{gathered}
$$

\section{Outage Probability}

Outage probability determines the probability of channel capacity under a certain rate which ensures data transmission without loss. The Outage Probability can be determined as:

$$
\mathrm{P}_{\text {out }}\left(C_{m}<R\right)=\int_{0}^{R} q\left(C_{m}\right) d C_{m}
$$

Where $C_{m}$ is the capacity of channel and $R$ is the certain Rate of capacity.

\subsection{MRC diversity}

Using (18), the outage probability of the capacity for MRC diversity is given by

$$
P_{\text {out }}^{M R C}\left(C_{m}<R\right)=\int_{0}^{R} e^{C_{m}} M \frac{\left(e^{C_{m}-1}\right)^{n_{R}-1}}{\left(n_{R}-1\right) !^{M} \gamma_{m_{o}}^{n_{R}}} e^{-\frac{e^{C_{m}-1}}{\gamma_{m_{o}}}} \times\left[\Gamma\left(n_{R}, \frac{e^{C_{m}-1}}{\gamma_{m_{o}}}\right)\right]^{M-1} d C_{m}
$$

Using the identity $\Gamma(n, x)=(n-1) ! e^{-x} \sum_{m=0}^{n-1} \frac{x^{m}}{m !}[7$, eq. (8.352.7)] 


$$
=\int_{0}^{R} e^{C_{m}} M \frac{\left(e^{C_{m}}-1\right)^{n_{R}-1}}{\left(n_{R}-1\right) !^{M} \gamma_{m_{o}}^{n_{R}}} e^{-\frac{e^{C_{m}-1}}{\gamma_{m_{o}}}}\left(n_{R}-1\right) !^{M-1} \times e^{-\frac{M-1}{\gamma_{m_{o}}}\left(e^{C_{m}}-1\right)} \sum_{v=0}^{\left(n_{R}-1\right)(M-1)} \beta\left(n_{R}, M-1\right)\left(\frac{e^{C_{m}}-1}{\gamma_{m_{o}}}\right)^{v} d C_{m}
$$

Where $\beta\left(n_{R}, M-1\right)$ is the coefficient of $\left(e^{C_{m}-1}\right)^{v}$ in the expansion of $\left[\sum_{v=0}^{n_{R}-1} \frac{1}{v !}\left(\frac{e^{C_{m}-1}}{\gamma_{m_{o}}}\right)^{v}\right]^{M-1}$. let, $e^{C_{m}-1}=z, e^{C_{m}} d C_{m}=d z$, when $C_{m}=0, z=0$ and when $C_{m}=R, z=e^{R}-1$

$$
\begin{gathered}
P_{\text {out }}^{M R C}=\sum_{v=0}^{\left(n_{R}-1\right)(M-1)} M \frac{\beta\left(n_{R}, M-1\right)}{\left(n_{R}-1\right) ! r_{m_{o}}^{n_{R}+v}} \int_{0}^{e^{R}-1} z^{n_{R}+v-1} e^{-\frac{M}{\gamma m_{o}} z} d z \\
P_{\text {out }}^{M R C}=\sum_{v=0}^{\left(n_{R}-1\right)(M-1)} M \frac{\beta\left(n_{R}, M-1\right)}{\left(n_{R}-1\right) ! r_{m_{o}}+v} \times\left[\frac{\left(n_{R}+v-1\right) !}{\left(\frac{M}{\gamma_{m}}\right)^{n_{R}+v}}-e^{-\frac{M}{\gamma_{m_{o}}}\left(e^{R}-1\right)} \sum_{k=0}^{n_{R}+v-1} \frac{\left(n_{R}+v-1\right) !}{k !} \frac{\left(e^{R}-1\right)^{k}}{\left(\frac{M}{\gamma_{m_{o}}}\right)^{n_{R}+v-k}}\right]
\end{gathered}
$$

\subsection{SC Diversity}

Using (18), the outage probability of the capacity for SC diversity is given by

$$
P_{\text {out }}^{S C}=\int_{0}^{R} e^{C_{m}} M \frac{n_{R}}{\gamma_{m_{o}}}\left[1-e^{-\frac{e^{C_{m-1}}}{\gamma_{m_{o}}}}\right]^{n_{R}-1} e^{-\frac{e^{C_{m}-1}}{\gamma_{m_{o}}}} \times\left[1-\left(1-e^{-\frac{e^{C_{m}-1}}{\gamma_{m_{o}}}}\right)^{n_{R}}\right]^{M-1} d C_{m}
$$

Using the identity, $(\mathrm{a}+\mathrm{x})^{\mathrm{n}}=\sum_{k=0}^{n}\left(\begin{array}{l}n \\ k\end{array}\right) x^{k} a^{n-k}$ of [7, eq. (1.111)], we have

$$
P_{\text {out }}^{S C}=\sum_{l=0}^{n_{R-1}} \sum_{w=0}^{M-1} \sum_{u=0}^{w . n_{R}} \frac{M n_{R}}{\gamma_{m_{o}}}(-1)^{l+u+w}\left(\begin{array}{c}
n_{R}-1 \\
l
\end{array}\right) \times\left(\begin{array}{c}
M-1 \\
w
\end{array}\right)\left(\begin{array}{c}
n_{R} w \\
u
\end{array}\right) \int_{0}^{R} e^{C_{m}} e^{-(k+l+1) \frac{e^{C_{m}}-1}{\gamma_{m_{o}}}} d C_{m}
$$

Let, $e^{C_{m}-1}=z, e^{C_{m}} d C_{m}=d z$, When $C_{m}=0, z=0$ And when $C_{m}=R, z=e^{R}-1$

$$
\begin{aligned}
P_{\text {out }}^{S C}=\sum_{l=0}^{n_{R}-1} \sum_{w=0}^{M-1} \sum_{u=0}^{w \cdot n_{R}} \frac{M n_{R}}{\gamma_{m_{o}}}(-1)^{l+u+w}\left(\begin{array}{c}
n_{R}-1 \\
l
\end{array}\right) \times\left(\begin{array}{c}
M-1 \\
w
\end{array}\right)\left(\begin{array}{c}
n_{R} w \\
u
\end{array}\right) \int_{0}^{e^{R}-1} e^{-\frac{(k+l+1)}{\gamma_{m_{o}}}} d z \\
=\sum_{l=0}^{n_{R-1}} \sum_{w=0}^{M-1} \sum_{u=0}^{w . n_{R}} \frac{M n_{R}}{\gamma_{m_{o}}}(-1)^{l+u+w}\left(\begin{array}{c}
n_{R}-1 \\
l
\end{array}\right) \times\left(\begin{array}{c}
M-1 \\
w
\end{array}\right)\left(\begin{array}{c}
n_{R} w \\
u
\end{array}\right) \frac{\gamma_{m_{0}}}{k+l+1}\left(1-e^{\frac{k+l+1}{\gamma_{m_{0}}\left(1-e^{R}\right)}}\right)
\end{aligned}
$$

\section{CCDF Of Capacity}

The CCDF of capacity determines the probability of the capacity being greater than the target rate given by

$$
C C D F=\int_{R}^{\infty} q\left(C_{m}\right) d C_{m}
$$

\subsection{MRC diversity}

Using (21), the CCDF of the capacity for MRC diversity is given by:

$$
C C D F_{C}{ }^{M R C}=\int_{R}^{\infty} e^{C_{m}} M \frac{\left(e^{C_{m}}-1\right)^{n_{R}-1}}{\left(n_{R}-1\right) ! !^{M} \gamma_{m_{o}}^{n_{R}}} e^{-\frac{e^{C_{m}-1}}{\gamma_{m_{o}}}} \times\left[\Gamma\left(n_{R}, \frac{e^{C_{m}}-1}{\gamma_{m_{o}}}\right)\right] d C_{m}
$$

Using the identity $\Gamma(n, x)=(n-1) ! e^{-x} \sum_{m=0}^{n-1} \frac{x^{m}}{m !}$ [7, eq. (8.352.7)]

$C C D F_{C}{ }^{M R C}=\int_{R}^{\infty} e^{C_{m}} M \frac{\left(e^{c_{m}}-1\right)^{n_{R}-1}}{\left(n_{R}-1\right) !^{M} \gamma_{m_{o}}^{n_{R}}} e^{-\frac{e^{C_{m}-1}}{\gamma m_{o}}}\left(n_{R}-1\right) !^{M-1} \times e^{-\frac{M-1}{\gamma_{m_{o}}}\left(e^{C_{m}}-1\right)} \sum_{v=0}^{\left(n_{R}-1\right)(M-1)} \beta\left(n_{R}, M-1\right)\left(\frac{e^{C_{m}-1}}{\gamma_{m_{o}}}\right)^{v} d C_{m}$

Where $\beta\left(n_{R}, M-1\right)$ is the coefficient of $\left(e^{C_{m}-1}\right)^{v}$ in the expansion of $\left[\sum_{v=0}^{n_{R}-1} \frac{1}{v !}\left(\frac{e^{C_{m}-1}}{\gamma_{m_{o}}}\right)^{v}\right]^{M-1}$.

Let, $e^{C_{m}-1}=z, e^{C_{m}} d C_{m}=d z$, when $C_{m}=R, z=e^{R}-1$ and when $C_{m}=\infty, z=\infty$

$$
C C D F_{C}{ }^{M R C}=\sum_{v=0}^{\left(n_{R}-1\right)(M-1)} M \frac{\beta\left(n_{R}, M-1\right)}{\left(n_{R}-1\right) ! \gamma_{m_{o}}^{n_{R}+v}} \int_{e^{R}-1}^{\infty} z^{n_{R}+v-1} e^{-\frac{M}{\gamma_{m_{o}}} z} d z
$$

$C C D F_{C}{ }^{M R C}=\sum_{v=0}^{\left(n_{R}-1\right)(M-1)} M \frac{\beta\left(n_{R}, M-1\right)}{\left(n_{R}-1\right) ! \gamma_{m_{o}}^{n_{R}+v}} \times\left[\frac{\left(n_{R}+v-1\right) !}{\left(\frac{M}{\gamma_{o}}\right)^{n_{R}+v}}-e^{-\frac{M}{\gamma_{m_{o}}}\left(e^{R}-1\right)} \sum_{k=0}^{n_{R}+v-1} \frac{\left(n_{R}+v-1\right) !}{k !} \frac{\left(e^{R}-1\right)^{k}}{\left(\frac{M}{\gamma_{o}}\right)^{n_{R}+v-k}}\right]$

\subsection{SC Diversity}

Using (21), the CDF of the capacity for SC diversity is given by:

$$
C C D F_{C} S C=\int_{R}^{\infty} e^{C_{m}} M \frac{n_{R}}{\gamma_{m_{o}}}\left[1-e^{-\frac{e^{C_{m}-1}}{\gamma_{m_{o}}}}\right]^{n_{R}-1} e^{-\frac{e^{C_{m}-1}}{\gamma_{m_{o}}}} \times\left[1-\left(1-e^{-\frac{e^{C_{m}-1}}{\gamma_{m_{o}}}}\right)^{n_{R}}\right]^{M-1} d C_{m}
$$

Using the identity, $(\mathrm{a}+\mathrm{x})^{\mathrm{n}}=\sum_{k=0}^{n}\left(\begin{array}{l}n \\ k\end{array}\right) x^{k} a^{n-k}$ of [7, eq. (1.111)], we have:

$$
C C D F_{C}{ }^{S C}=\sum_{l=0}^{n_{R}-1} \sum_{w=0}^{M-1} \sum_{u=0}^{w \cdot n_{R}} \frac{M n_{R}}{\gamma_{m_{o}}}(-1)^{l+u+w}\left(\begin{array}{c}
n_{R}-1 \\
l
\end{array}\right) \times\left(\begin{array}{c}
M-1 \\
w
\end{array}\right)\left(\begin{array}{c}
n_{R} w \\
u
\end{array}\right) \int_{R}^{\infty} e^{C_{m}} e^{-(k+l+1) \frac{e^{c_{m}}-1}{\gamma_{m}}} d C_{m}
$$

Let, $e^{C_{m}-1}=z, e^{C_{m}} d C_{m}=d z$, when $C_{m}=R, z=e^{R}-1$ and when $C_{m}=\infty, z=\infty$ 


$$
\begin{aligned}
& C C D F_{C} S C=\sum_{l=0}^{n_{R}-1} \sum_{w=0}^{M-1} \sum_{u=0}^{w \cdot n_{R}} \frac{M n_{R}}{\gamma_{m_{o}}}(-1)^{l+u+w}\left(\begin{array}{c}
n_{R}-1 \\
l
\end{array}\right) \times\left(\begin{array}{c}
M-1 \\
w
\end{array}\right)\left(\begin{array}{c}
n_{R} w \\
u
\end{array}\right) \int_{e^{R}-1}^{\infty} e^{-\frac{(k+l+1)}{\gamma_{m_{o}}} z} d z \\
& =\sum_{l=0}^{n_{R-1}} \sum_{w=0}^{M-1} \sum_{u=0}^{w . n_{R}} \frac{M n_{R}}{\gamma_{m_{o}}}(-1)^{l+u+w}\left(\begin{array}{c}
n_{R}-1 \\
l
\end{array}\right) \times\left(\begin{array}{c}
M-1 \\
w
\end{array}\right)\left(\begin{array}{c}
n_{R} w \\
u
\end{array}\right) \frac{\gamma_{m_{0}}}{k+l+1}\left(e^{\frac{k+l+1}{\gamma_{m_{0}}}\left(1-e^{R}\right)}\right)
\end{aligned}
$$

\section{Numerical Results}

The analytical expressions of section 4 and section 5 are justified in this section. Fig. 2 graphically represents the Outage Probability as a function of target rate of capacity for both MRC and SC diversity combining techniques. The result is shown for different number of receiving antennas.The outage probability is lower in case of MRC. In both cases of MRC and SC the outage probability decreases as the number of receiving antenna increases but the decrement is larger for MRC diversity.

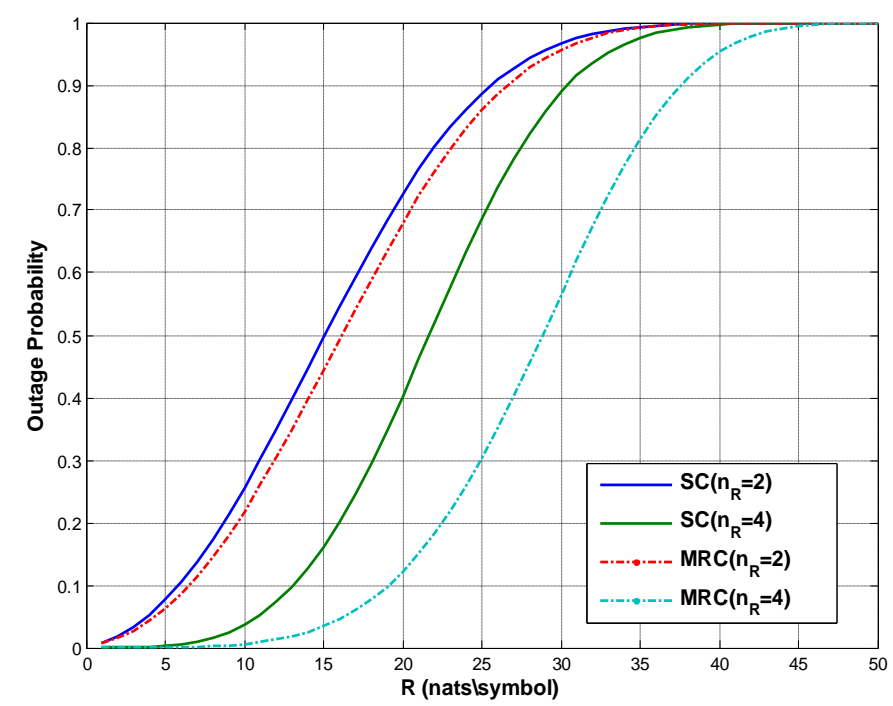

Figure 2: Outage Probability versus target rate for selected SNR of channel

Fig.3 represents the CCDF of capacity as a function of target rate of capacity for both MRC and SC diversity combining techniques. The result is varied for different number of receiving antennas. The CCDF is higher in case of MRC diversity. The CCDF increases when the number of receiving antenna increases in both cases but the effect of antenna is larger in case of MRC diversity

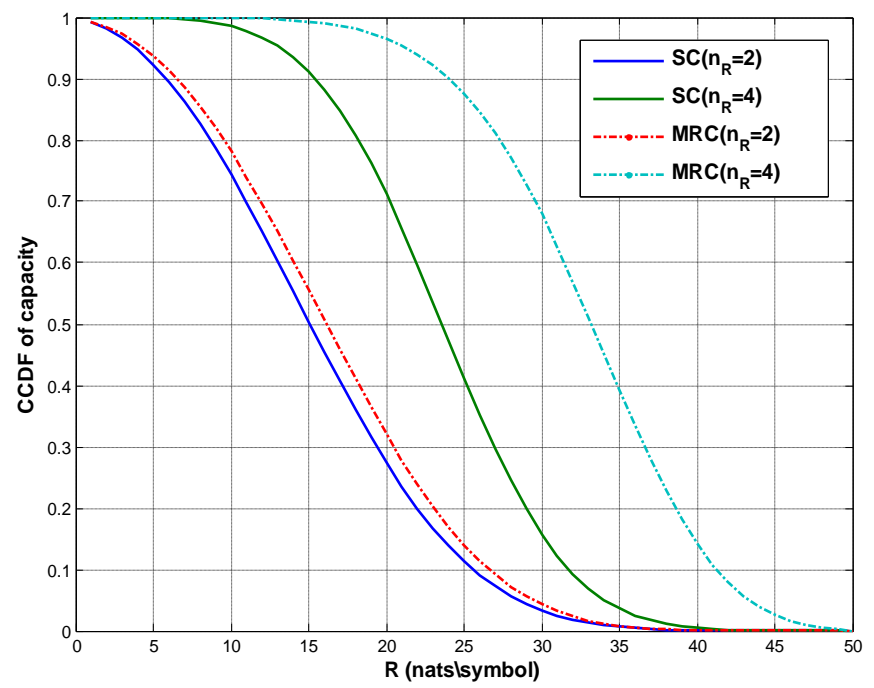

Figure 3: CCDF of capacity versus target rate for selected SNR of channel 


\section{Conclusion}

We consider a multicasting scenario and focus on outage performance. We derived closed form expressions of Outage Probability and CCDF of multicast capacity and finally graphically represented the analytical expressions. From the numerical result we can conclude that MRC diversity shows better performance than SC diversity. Moreover, the effect of increasing the number of receiving antenna is more prominent in case MRC diversity than SC diversity. This result leads to a conclusion that MRC diversity technique is more suitable than SC diversity technique for multicasting communication.

\section{References}

[1]. Andrea Goldsmith, WIRELESS COMMUNICATIONS, $7^{\text {th }}$ Edition, New York, Cambridge University Press, 2005.

[2]. D. G. Brennan, Linear Diversity Combining Techniques, Proc. IEEE, Vol. 91, Pages: 331-356, $29^{\text {th }}$ April, 2003.

[3]. Luu Pham Tuyen, Vo Nguyen Quoc Bau, Comparison of Diversity Combining Techniques for MIMO Systems, Proc. 17 ${ }^{\text {th }}$ Asia Pacific Conference on Communications, 2-5 October, 2011.

[4]. Jorg Nuchell, Hendrick Hoffmann, Moritz Schack, Thomas kurner, Linear Diversity Combining Techniques Employed Car-to-X Communication Systems, Proc. 73 ${ }^{\text {rd }}$ IEEE Vehicular Technology Conference (VTC Spring), 15-18 May, 2011.

[5]. A. Arokiamarie Johnsi, V. Saminadan, Performance for Diversity Combining Techniques for FSO MIMO System, Proc. International Conference on Communications and Signal Processing (ICCSP), 3-5 April, 2013.

[6]. Sari Khatalin, John Fonseka, Capacity of Correlated Nakagami-m Fading Channels with Diversity Combining Techniques, IEEE Transactions on Vehicular Technology, pp. 142-150, February, 2006.

[7]. I. S. Gradshteyn and I. M. Ryzhik, Table of Integrals, Series, and Products, 7th ed. San Diego, CA: Academic, 2007. 